\title{
Chinese Herbal Tanshinol Prevents Osteoporosis via Coordinately Promoting Osteogenesis and Inhibiting Osteoclastogenesis
}

tailin wu

shenzhen university general hospital

jianchao wang

The Third People's Hospital of Shenzhen

jianzhou luo

Shenzhen University Health Science Center

haitao lin

shenzhen university general hospital

fei wang

Shenzhen University General Hospital

yanzhe wei

Shenzhen University General Hospital

chunguang duan ( $1910244002 @ e m a i l . s z u . e d u . c n$ )

Shenzhen University General Hospital https://orcid.org/0000-0003-0153-279X

huiren tao

Shenzhen University General Hospital

\section{Research}

Keywords: Tanshinol, osteogenesis, osteoclastogenesis, osteoporosis, Wnt, Akt

Posted Date: January 7th, 2021

DOI: https://doi.org/10.21203/rs.3.rs-139701/v1

License: (1) (1) This work is licensed under a Creative Commons Attribution 4.0 International License. Read Full License 


\section{Abstract}

Background: Osteoporosis severely affects patients' life quality due to increased risks of fragility fractures. Tanshinol is a primary water-soluble compound purified from the Chinese herb Salvia Miltiorrhiza, which exhibits potent antioxidant and anti-inflammatory properties. However, whether Tanshinol functions in preventing and protecting osteoporosis remains unknown. Thus, current study proposed to systematically investigate the protective effects and the underlying mechanisms of Tanshinol in bone marrow mesenchymal stem cells (BM-MSCs) and ovariectomized (OVX) mice model.

Materials and methods: Different concentrations of Tanshinol were given to induce differentiation of BMMSCs respectively, and detected the expression of key markers of osteoclast and osteogenesis. The C57BL/ 6 mice were divided into control group, model group, low ( $80 \mathrm{mg} / \mathrm{kg} \times$ day, i.g), medium (160 $\mathrm{mg} / \mathrm{kg} \times$ day, i.g) and high ( $240 \mathrm{mg} / \mathrm{kg} \times$ day, i.g) concentrations Tanshinol groups. After 6 weeks treatment of Tanshinol, mice distal femurs were taken to measure bone mineral density (BMD) and three dimension parameters.

Results: In the present study, we for the first time showed that Tanshinol could promote osteogenesis in the mouse BM-MSCs, as seen from the increase of the osteogenic markers such as ALP activity and collagen I. Meanwhile, Tanshinol inhibited the RANKL induced osteoclastogenesis in the bone marrow monocytes (BMMs). Animal studies showed that oral delivery of Tanshinol could attenuate in ovariectomy induced osteoporosis. Molecularly, Tanshinol activated Wnt signal pathway in MSCs while inhibited Akt in BMMs, suggesting these pathways might be involved in the osteoporosis protective role of Tanshinol.

Conclusions: our study has revealed a potential application of Salvia miltiorrhiza derivative Tanshinol in the treatment of osteoporosis.

\section{Background}

Osteoporosis is a systemic skeletal disease characterized by a progressive loss of bone mass and microarchitectural deterioration of bone tissue, and therefore leads to increased risk of fragility fractures[1]. Current treatments of osteoporosis include changing lifestyles, taking orthopedic drugs (bone anti-resorption and bone formation therapy), and invasive surgeries. However, these treatment options are not long lasting and can lead to complications after post-surgical life [2]. Numerous researches have demonstrated that oxidative stress plays a crucial role in the development of osteoporosis [3-5]. Decreased bone mineral density (BMD), a general feature of osteoporosis, was shown to be associated with higher oxidative stress index values and total plasma oxidant status in osteoporotic patients[6]. Bone loss can result either from enhanced osteoclast bone resorption or decreased osteoblast bone formation. Chronic exposure to oxidative stress and inflammation can result in the unbalance of the bone resorption and formation[7].Therefore, the application of antioxidant medicine may be new options for the prevention and cure of osteoporosis. 
It was previously reported that Tanshinol $(D(+) \beta-3,4$-dihydroxyphenyl lactic acid; or named Danshensu) might attenuate oxidative stress injury in many non-bone tissues and cells [8-11].Tanshinol can also increase osteogenesis in GC-treated larval zebrafish via scavenging ROS and stimulate the expression of osteoblast-specific genes[12]. Animal experiments also demonstrated that Tanshinol had beneficial effects to the Glucocorticoid-induced osteoporosis[13]. Although the potent antioxidant and antiinflammatory properties of Tanshinol may be of therapeutic potential for the treatment of different bone diseases, its potential effects on the postmenopausal osteoporosis remain elusive. In this study, we aimed to examine its effect of postmenopausal osteoporosis in an OVX mouse model, with underlying mechanisms during the osteogenic differentiation and osteoclastic activity explored.

\section{Materials And Methods}

\section{Isolation and culture of mouse BM-MSCs and osteoclasts.}

For isolation of mouse BM-MSCs, 4 week-old C57BL/6 mice (Fourth Military Medical University, Xi'an, China) were sacrificed. Aseptically detached tibias and femurs, trimmed away excess soft tissues, and washed marrow cavity with PBS at $4{ }^{\circ} \mathrm{C}$. Bone marrow cells were collected by centrifugation at $800 \times \mathrm{g}$ for 5 min and resuspended in $2 \mathrm{ml}$ Dulbecco's modified Eagle's medium (DMEM) medium plus 10\% fetal bovine serum (FBS) $(\mathrm{v} / \mathrm{v})$. The resuspended cells were seeded in a tissue culture flask for culture in a humidified $5 \% \mathrm{CO} 2$ incubator at $37^{\circ} \mathrm{C}$. Then changed the medium every 2 days, and removed the floating cells. Upon reached $80-90 \%$ confluent, the adherent cells were passaged by trypsinization and subcultured. Cells after three passages were prepared for experiments. And bone marrow cells were obtained from the long bones of 6 week-old C57BL/ 6 mice (Fourth Military Medical University, Xi'an, China). Bone marrow cells cultured in the presence of M-CSF (50 ng/ml, PeproTech, Inc., Rocky Hill, NJ, USA) for 3 days to generate the bone marrow derived macrophages (BMMs). To examine osteoclast formation, BMMs were treated with Tanshinol at different concentrations in the presence of M-CSF (50 ng/ml) and RANKL (100 ng/ml, PeproTech, Inc., Rocky Hill, NJ, USA) in 96 well culture plates (Corning, MA, USA). Cells were fixed and stained for tartrate resistant acid phosphatase (TRAP), a marker enzyme of osteoclasts. Experimental protocol involving animals was reviewed and approved by the Institutional Animal Care and Use Committee of the Fourth Military Medical University. All experiments were performed in accordance with the relevant guidelines and regulations of the Institutional Animal Care and Use Committee of the Fourth Military Medical University.

\section{Induction of osteoblast differentiation and Alizarin red-sulfate staining}

In the study, osteogenic differentiation of mouse BM-MSCs was induced by osteogenic medium (MUBMX90021, Cyagen, USA). To examine the osteogenesis of mouse BM-MSCs, cells were seeded into a 96-well plate at $\sim 80 \%$ confluent and the osteogenic medium added Tanshinol with different concentrations was changed every 2 days. After culturing 21 days, the cells were treated with Alizarin red-sulfate (ARS) staining. For Alizarin red-sulfate (ARS) staining, removed the medium, washed the cells with PBS for three times and fixed in $70 \%$ methanol $(\mathrm{v} / \mathrm{v})$ at room temperature for $30 \mathrm{~min}$. After washing three times with 
PBS, the cells were stained with $40 \mathrm{mM}$ Alizarin red-sulfate (Sigma-Aldrich) aqueous solution, $\mathrm{pH} 4.2$, for $20 \mathrm{~min}$ at room temperature using an orbital shaker at 100 rpm. The cells were further rinsed with PBS to remove nonspecific staining and allowed to dry completely.

\section{Cytotoxicity test}

MTT method was used to analysis the impact on BMMs. BMMs were seeded in 96-well plates with the density of $1 \times 10^{5} / \mathrm{ml}$, treated with Tanshinol at different concentrations. After 48 hours, $10 \mu \mathrm{L}(5 \mathrm{mg} / \mathrm{ml})$ MTT per hole were added and incubated for 4 hours at $37^{\circ} \mathrm{C}$. The cells were further added DMSO $150 \mu \mathrm{L}$ per hole and dissolved for 10 minutes and then measured OD value at $490 \mathrm{~nm}$ absorbance.

\section{Quantitative reverse transcription PCR (RT-PCR)}

Quantitative RT-PCR experiments were conducted according to routine protocols. Total RNA was extracted with Trizol reagent (Invitrogen, USA), and cDNA was synthesized with a cDNA synthesis kit (Clontech, USA). Target genes were amplified from the synthesized CDNA and quantified by densitometry. The relative gene expression level was normalized against the $\beta$-actin transcript level. Each value represented the average of three independent experiments. The following primers were used for the amplification of target genes:

ALP forward primer 5'-AACCCAGACACAAGCATTCC-3' and reverse primer 5'-GCCTTTGAGGTTTTTGGTCA3';

Collagen I forward primer 5'-ACAGCCGCTTCACCTACAGC-3' and reverse primer 5'TGCACTTTTGGTTTTTGGTCAT-3';

$\beta$-catenin forward primer 5'-GGCAACCAAGAAAGCAAG-3' and reverse primer 5'CTGAACAAGAGTCCCAAGGAG-3';

Axin2 forward primer 5'-TGACTCTCCTTCCAGATCCCA-3' and reverse primer TGCCCACACTAGGCTGACA3';

C-fos forward primer 5'-CGGGTTTCAACGCCGACTA-3' and reverse primer 5'-

TTGGCACTAGAGACGGACAGA-3';

CTSK forward primer 5'-GAAGAAGACTCACCAGAAGCAG-3'and reverse primer5'TCCAGGTTATGGGCAGAGATT-3';

TRAP forward primer 5'-CACTCCCACCCTGAGATTTGT-3' and reverse primer 5'CATCGTCTGCACGGTTCTG-3';

NFATc1 forward primer 5'-TGGGAGATGGAAGCAAAGACTGA-3' and reverse primer 5'CATTGGCAGGAAGGTACGTGA A-3'; 
$\beta$-actin forward primer 5'-CAATGTGGCCGAGGACTTTG-3' and reverse primer 5'-

CATTCTCCTTAGAGAGAAGTGG-3'.

\section{Western blotting analysis}

Proteins from osteoblasts and osteoclasts or the precursors with indicated treatments were extracted with ice-cold lysis buffer and centrifuged at $12,000 \mathrm{~g}$ for $10 \mathrm{~min}$., and the resultant supernatant assayed using BCA protein assay kit standardized to BSA. Equal amounts of total protein $(40 \mu \mathrm{g})$ were loaded, separated by $12 \%$ SDS-PAGE, and transferred to polyvinylidene fluoride (PVDF) membrane. The membranes were blocked in 5\% TBST fat free milk for 2 hours, briefly washed, hybrid antibodies and then quantified using Imaging System Analysis software (VersaDoc Mp5000; Bio-Rad). Expression of $\beta$-actin protein served as a control.

\section{Animals and Experimental Design}

7 week-old C57BL/6 mice were purchased from Experimental Animal Research Center, the Fourth Military Medical University (Xi'an, China). The animals were maintained in individually ventilated cages (IVC) system ( $12 \mathrm{~h}$ light/dark cycle, $20.3-23.1^{\circ} \mathrm{C}$ and $40-50 \%$ humidity) during the experiment cycle and fed with standard laboratory food and water ad libitum. The experimental animals were divided into five groups of six mice each.

Group I (Control group): sham operation group received normal saline (160 mg/ kg×day, i.g) for a period of 6 weeks;

Group II (Model group): OVX group received normal saline (160 mg/ kg×day, i.g) for a period of 6 weeks;

Group III (Tanshinol low-concentration group): mice treated with bilateral ovariectomy received Tanshinol $(80 \mathrm{mg} / \mathrm{kgday}, \mathrm{i} . \mathrm{g})$ for a period of 6 weeks;

Group IV (Tanshinol medium-concentration group): mice treated with bilateral ovariectomy received Tanshinol (160 mg/ kg×day, i.g) for a period of 6 weeks;

Group V (Tanshinol high-concentration group): mice treated with bilateral ovariectomy received Tanshinol $(240 \mathrm{mg} / \mathrm{kgday}, \mathrm{i} . \mathrm{g})$ for a period of 6 weeks.

At the end of the experimental period, mice were sacrificed by cervical decapitation. We used micro computed tomography (microCT) to examine the bone mineral density (BMD) and three-dimensional architecture parameters in trabecular bone of the distal femur.

\section{Statistical analysis}

All experiments were independently repeated for at least three times. All data were shown as mean \pm SEM. ANOVA was used for statistical analysis, and $\mathrm{P}<0.05$ was considered significant. 


\section{Results}

\section{Effects of Tanshinol on the osteogenesis of mouse BM-MSCs}

ALP gene expression was examined by qPCR at day 7 after the administration of Tanshinol at different concentrations. The statistical analysis of three independent experiments showed that ALP gene expression was markedly promoted by Tanshinol at 0.1,1 and $10 \mu \mathrm{M}$ (Fig.1b). Similarly, the effect of Tanshinol on the osteogenesis at the late stage was examined at day 21 by ARS staining (Fig.1a), indicating that osteogenesis was improved at the early and late stage by Tanshinol. Then expression levels of osteogenic and Wnt pathway-related marker genes were examined at day 21 with RT-PCR and western blot. Collagen I were upregulated by Tanshinol at 0.1,1 and $10 \mu \mathrm{M}$ (Fig. $1 \mathrm{~b}$ and $1 \mathrm{c}$ ), and three independent experiments confirmed that such upregulation was significant $(p<0.05)$. Similarly, Wnt pathway-related regulators $\beta$-catenin and Axin 2 increased as well as the activity of Tcf transcription factors responsible for bone formation (Fig. 1d), suggesting Tanshinol activated Wnt pathway via both $\beta$ catenin expression and nuclear translocation.

\section{Effects of Tanshinol on RANKL-induced Osteoclastogenesis}

For detection of the effects of Tanshinol on the RANKL-induced osteoclastogenesis, BMMs were treated with Tanshinol at different concentrations $(0,25$ and $50 \mu \mathrm{M})$ in the presence of M-CSF and RANKL. Results showed that the numbers of the TRAP positive, multinucleated mature osteoclasts were significantly declined and dose-dependent (Fig.2a).In order to determine the influence of Tanshinol on the osteoclasts' proliferation and survival, BMMs, the osteoclast precursors, were determined by MTT analysis. And BMMs osteoclastogenesis differentiation were inhibited by Tanshinol even at the the dose of $0.1 \mu \mathrm{M}$ (Fig.2b). To illustrate the mechanism of the inhibition of the osteoclast differentiation, we detected the known signal transduction pathway involved, including p38MAPK, ERK, JNK, Akt. After the administration of Tanshinol for 2 hours followed by RANKL for 5 minutes, phosphorylated Akt level was significantly decreased but the phosphorylation levels of p38 MAPK, JNK, ERK did not change significantly (Fig.2C).The results indicated that Tanshinol might inhibit RANKL-induced osteoclastogenesis via downregulation of Akt phosphorylation. Since both c-fos and NFATc1 play critical role in osteoclastogenesis tweaking the osteoclasts' specific genes expression, we next examined the effect of Tanshinol on BMMs at 1, 2 day induced by RANKL. RT-PCR analysis revealed that at day 1, the mRNA levels of c-fos were significantly downregulated and c-fos, TRAP and CTSK were significantly decreased VS control at day 2(Fig.2d). However the mRNA levels of NFATc1 (data were not shown) were not significantly changed.

\section{Tanshinol protects OVX mice from osteoporosis}

We used micro CT to examine the BMD and three dimensional architecture parameters in trabecular bone of the distal femur after the administration of Tanshinol at different concentrations in 7 week-old C57BL/6 mice treated with ovariectomy (OVX). The statistical analysis for the micro CT data showed that effect of Tanshinol was statistically significant when compared with control model group for all the 
variables we examined (BMD, trabecular number, trabecular space and relative bone volume ) (Fig.3a, 3b and 3c). And no statistical significance was found between Tanshinol with $160 \mathrm{mg} / \mathrm{kg} \times \mathrm{d}$ group with the model group in the trabecular space and BV/TV. Meanwhile, no statistical significance were found between different concentrations groups.

\section{Discussion}

To our knowledge, this is the first report that Tanshinol can coordinately promote osteogenesis while inhibit osteoclastogenesis, possibly via Wnt/AkT pathways, and we also validated that Tanshinol could attenuate osteoporosis in mouse model induced by ovariectomy (OVX). As such these findings may help enable the development of Tanshinol-based bone anabolic therapies.

Tanshinol, an active ingredient of traditional Chinese medicinal herb Salvia Miltiorrhiza, has been widely used in eastern Asia in the treatment of cardiovascular[14-16], cerebrovascular[17] syndromes, liver injury[18, 19], chronic kidney diseases[20] and anxiolytic-like effect[21] due to its potent antioxidant and anti-inflammatory properties. Previous literatures reported that Salvia Miltiorrhiza, has the action of increasing the strength of fracture healing site [22] .The aqueous extract of Salvia Miltiorrhiza, can prevent the osteoporosis induced by glucocorticoid [23], indicating Tanshinol plays a critical role in the curing of osteoporosis. And Tanshinol attenuates osteoporosis mediated by oxidative stress via FoxO/Wnt signaling [24].

In the present study, Tanshinol was found to alter the proliferation of mouse BM-MSCs significantly. Such promoting effect of Tanshinol on cell proliferation was also previously reported in many other cell types [24-26]. We also verified the promoting effect was achieved by activating the $\mathrm{Wnt} / \beta$-catenin/Tcf signal pathway. For the inhibition of osteoclastogenesis, $0.1 \mu \mathrm{M}$ Tanshinol did not cause obvious cytotoxic reaction, while showed obvious inhibitory effects on osteoclastogenesis in BMMs. Further mechanism studies revealed decrease of phosphorylated Akt might be involved, as activation of ERK and AKT pathway plays critical role in the osteoclasts differentiation [27-29]. Notably, our study here was contradicted to previous reports that Tanshinol inhibited apoptosis by acting PI3K/Akt pathway [15], which might be explained by the concentration and duration of Tanshinol treatment, cell types, and culturing conditions. The animal experiments confirmed the prevention and cure effect of Tanshinol on osteoporosis in OVX mouse model. Interestingly, the efficacy was not significantly different among the 80,160 and $240 \mathrm{mg} / \mathrm{kg} \times \mathrm{d}$ groups, which might be due to the lowest dose of $80 \mathrm{mg} / \mathrm{kg} \times \mathrm{d}$ was enough to prevent osteoporosis [24], suggesting a safe application of Tanshinol in osteoporosis[30].

\section{Conclusions}

In summary, our study here established that Tanshinol activated Wnt signal pathway in MSCs while inhibited Akt in BMMs, resulting in coordinately promoting osteogenesis and inhibiting osteoclastogenesis. Furthermore, Tanshinol prevented OVX mice from osteoporosis in vivo. All of these 
data suggest a potential application of Salvia Miltiorrhiza, derivative Tanshinol in the treatment of osteoporosis.

\section{Abbreviations}

ALP, alkaline phosphatase; ARS, alizarin red-sulfate; BMD, bone mineral density; BMMs, bone marrow monocytes; BM-MSCs, bone marrow mesenchymal stem cells; Collagen I, type I collagen; i.g, intragastrically; M-CSF, macrophage colony-stimulating factor; MSCs, mesenchymal stem cells; OVX, ovariectomized; RANKL, receptor activator of nuclear factor (NF)-kappa B ligand; TCF, T-cell-specific transcription factor; TRAP, tartrate resistant acid phosphatase.

\section{Declarations}

Tailin Wu, Jianchao Wang, Jianzhou Luo, Haitao Lin, Fei Wang,Yanzhe Wei, Chunguang Duan and Huiren Tao declare that they have no conflict of interest.

\section{Ethics approval and consent to participate}

All animal experiments were approved by the Institutional Animal Ethics Committee of the Fourth Military Medical University.

\section{Consent for publication}

Not applicable.

\section{Availability of data and materials}

The datasets used and/or analyzed during the current study are available from the corresponding author on reasonable request.

\section{Competing interests}

The authors have no competing interests.

\section{Funding}

This study was funded by Shenzhen Technical Project (Grant No. JCYJ20180305124242438),National Natural Science Foundation of China (81970761), Sanming Project of Medicine in Shenzhen (SZSM201911011) and Natural Science Foundation of Guangdong Province (2020A151501726).

\section{Authors' contributions}


Tailin Wu, Jianchao Wang, Jianzhou Luo and Haitao Lin performed the experiments and wrote the manuscript, Fei Wang and Yanzhe Wei analyzed the data, Chunguang Duan and Huiren Tao conceived the idea and designed the study.

\section{Acknowledgements}

We would like to thank guodong yang for the preparation of documentations and assistance with the data analyses.

\section{References}

1. Rachner TD, Khosla S, Hofbauer LC: Osteoporosis: now and the future. The Lancet 2011;377:12761287.

2. Wei D, Jung J, Yang H, Stout DA, Yang L: Nanotechnology Treatment Options for Osteoporosis and Its Corresponding Consequences. Current osteoporosis reports 2016

3. Almeida M: Unraveling the role of FoxOs in bone-insights from mouse models. Bone 2011;49:319327.

4. Liao L, Su X, Yang X, Hu C, Li B, Lv Y, Shuai Y, Jing H, Deng Z, Jin Y: TNF-alpha Inhibits FoxO1 by Upregulating miR-705 to Aggravate Oxidative Damage in Bone Marrow-Derived Mesenchymal Stem Cells during Osteoporosis. Stem cells (Dayton, Ohio) 2016;34:1054-1067.

5. Wauquier F, Leotoing L, Coxam V, Guicheux J, Wittrant Y: Oxidative stress in bone remodelling and disease. Trends in molecular medicine 2009;15:468-477.

6. Altindag O, Erel O, Soran N, Celik H, Selek S: Total oxidative/anti-oxidative status and relation to bone mineral density in osteoporosis. Rheumatology international 2008;28:317-321.

7. Redlich K, Smolen JS: Inflammatory bone loss: pathogenesis and therapeutic intervention. Nat Rev Drug Discov 2012;11:234-250.

8. Zhang H, Xu Y, Wang J, Zhang K, Yi B, Liu Y, Cai X: [Effect of Danshensu on fibronectin and collagen1 secretion induced by high glucose in human peritoneal mesothelial cells]. Zhong Nan Da Xue Xue Bao Yi Xue Ban 2011;36:44-50.

9. Yu J, Wang L, Akinyi M, Li Y, Duan Z, Zhu Y, Fan G: Danshensu protects isolated heart against ischemia reperfusion injury through activation of Akt/ERK1/2/Nrf2 signaling. International journal of clinical and experimental medicine 2015;8:14793-14804.

10. Chen Y, Liu Z, Zhou F, Zhao H, Yang Q, Li H, Sun J, Wang S: Evaluating Pharmacological Effects of Two Major Components of Shuangdan Oral Liquid: Role of Danshensu and Paeonol in Diabetic Nephropathy Rat. Biomolecules \& therapeutics 2016;24:536-542.

11. Zhao GR, Zhang HM, Ye TX, Xiang ZJ, Yuan YJ, Guo ZX, Zhao LB: Characterization of the radical scavenging and antioxidant activities of danshensu and salvianolic acid B. Food Chem Toxicol 2008;46:73-81. 
12. Luo S, Yang Y, Chen J, Zhong Z, Huang H, Zhang J, Cui L: Tanshinol stimulates bone formation and attenuates dexamethasone-induced inhibition of osteogenesis in larval zebrafish. Journal of Orthopaedic Translation 2016;4:35-45.

13. Yang Y, Su Y, Wang D, Chen Y, Liu Y, Luo S, Wu T, Cui L: Tanshinol Rescues the Impaired Bone Formation Elicited by Glucocorticoid Involved in KLF15 Pathway. Oxid Med Cell Longev 2016;2016:1092746.

14. Li H, Song F, Duan LR, Sheng JJ, Xie YH, Yang Q, Chen Y, Dong QQ, Zhang BL, Wang SW: Paeonol and danshensu combination attenuates apoptosis in myocardial infarcted rats by inhibiting oxidative stress: Roles of Nrf2/HO-1 and PI3K/Akt pathway. Sci Rep 2016;6:23693.

15. Yin Y, Guan Y, Duan J, Wei G, Zhu Y, Quan W, Guo C, Zhou D, Wang Y, Xi M, Wen A: Cardioprotective effect of Danshensu against myocardial ischemia/reperfusion injury and inhibits apoptosis of H9c2 cardiomyocytes via Akt and ERK1/2 phosphorylation. Eur J Pharmacol 2013;699:219-226.

16. Pan LL, Wang J, Jia YL, Zheng HM, Wang Y, Zhu YZ: Asymmetric synthesis and evaluation of danshensu-cysteine conjugates as novel potential anti-apoptotic drug candidates. International journal of molecular sciences 2015;16:628-644.

17. Hu J, Li YL, Li ZL, Li H, Zhou XX, Qiu PC, Yang Q, Wang SW: Chronic supplementation of paeonol combined with danshensu for the improvement of vascular reactivity in the cerebral basilar artery of diabetic rats. Int J Mol Sci 2012;13:14565-14578.

18. Ren R, Wang T, Jiang N, Liu T, Du Y, Li C, Zhang L, Fu F: Protective effects of Danshensu on liver injury induced by omethoate in rats. Toxicol Mech Methods 2010;20:510-514.

19. Zhou X, Cheung CM, Yang JM, Or PM, Lee WY, Yeung JH: Danshen (Salvia miltiorrhiza) water extract inhibits paracetamol-induced toxicity in primary rat hepatocytes via reducing CYP2E1 activity and oxidative stress. The Journal of pharmacy and pharmacology 2015;67:980-989.

20. Guan Y, Wu XX, Duan JL, Yin Y, Guo C, Wei G, Wang YH, Zhu YR, Weng Y, Xi MM, Wen AD: Effects and Mechanism of Combination of Rhein and Danshensu in the Treatment of Chronic Kidney Disease. The American journal of Chinese medicine 2015;43:1381-1400.

21. Kwon G, Kim HJ, Park SJ, Lee HE, Woo H, Ahn YJ, Gao Q, Cheong JH, Jang DS, Ryu JH: Anxiolytic-like effect of danshensu [(3-(3,4-dihydroxyphenyl)-lactic acid)] in mice. Life sciences 2014;101:73-78.

22. Fu S, Du N, Shi W: [Biomechanical experimental study on effective fraction of radix salviae miltiorrhizae on healing of bone fracture]. Zhongguo Zhong Xi Yi Jie He Za Zhi 1999;19:106-107.

23. Cui L, Liu YY, Wu T, Ai CM, Chen HQ: Osteogenic effects of D+beta-3,4-dihydroxyphenyl lactic acid (salvianic acid A, SAA) on osteoblasts and bone marrow stromal cells of intact and prednisonetreated rats. Acta Pharmacol Sin 2009;30:321-332.

24. Yang Y, Su Y, Wang D, Chen Y, Wu T, Li G, Sun X, Cui L: Tanshinol attenuates the deleterious effects of oxidative stress on osteoblastic differentiation via Wnt/FoxO3a signaling. Oxid Med Cell Longev 2013;2013:351895.

25. Chen YS, Lee SM, Lin YJ, Chiang SH, Lin CC: Effects of Danshensu and Salvianolic Acid B from Salvia miltiorrhiza Bunge (Lamiaceae) on cell proliferation and collagen and melanin production. 
Molecules 2014;19:2029-2041.

26. Gao H, Li L, Li L, Gong B, Dong P, Fordjour PA, Zhu Y, Fan G: Danshensu Promotes Cholesterol Efflux in RAW264.7 Macrophages. Lipids 2016;51:1083-1092.

27. Kawamura N, Kugimiya F, Oshima Y, Ohba S, Ikeda T, Saito T, Shinoda Y, Kawasaki Y, Ogata N, Hoshi K, Akiyama T, Chen WS, Hay N, Tobe K, Kadowaki T, Azuma Y, Tanaka S, Nakamura K, Chung UI, Kawaguchi H: Akt1 in osteoblasts and osteoclasts controls bone remodeling. PloS one 2007;2:e1058.

28. Moon JB, Kim JH, Kim K, Youn BU, Ko A, Lee SY, Kim N: Akt induces osteoclast differentiation through regulating the GSK3beta/NFATc1 signaling cascade. Journal of immunology 2012;188:163169.

29. Qu X, Zhai Z, Liu X, Li H, Ouyang Z, Wu C, Liu G, Fan Q, Tang T, Qin A, Dai K: Dioscin inhibits osteoclast differentiation and bone resorption though down-regulating the Akt signaling cascades. Biochem Biophys Res Commun 2014;443:658-665.

30. Gao Y, Liu Z, Li G, Li C, Li M, Li B: Acute and subchronic toxicity of danshensu in mice and rats. Toxicology mechanisms and methods 2009;19:363-368.

\section{Figures}


a

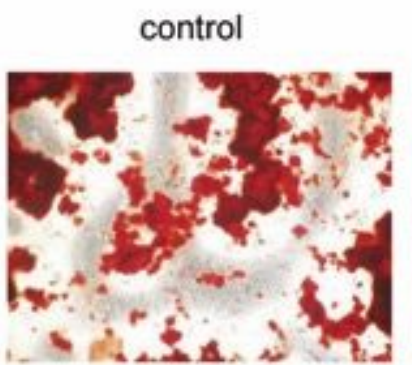

$\operatorname{Tan} 0.1 \mu \mathrm{M}$

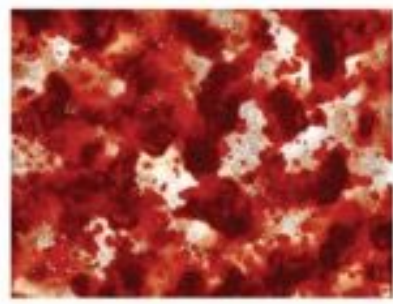

$\operatorname{Tan} 1 \mu \mathrm{M}$

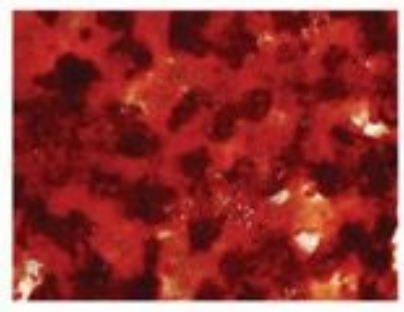

C b

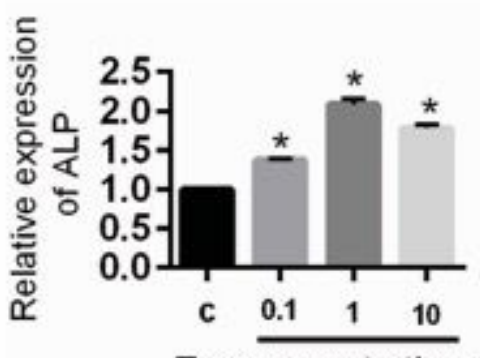

d

Tan concentrations

( $\mu \mathrm{M})$

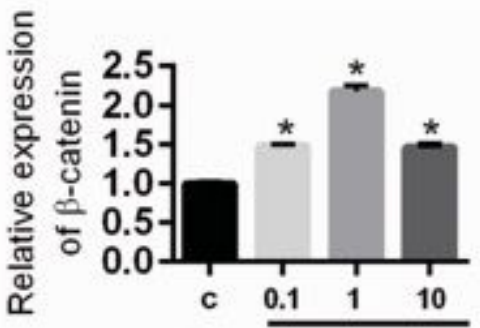

Tan concentrations

$(\mu \mathrm{M})$
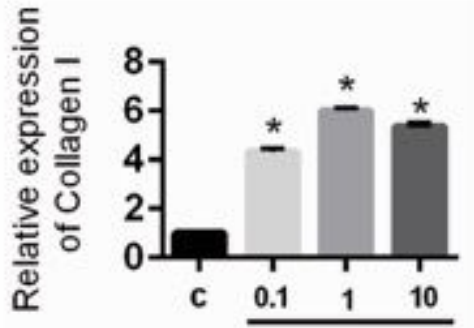

Tan concentrations

$(\mu \mathrm{M})$

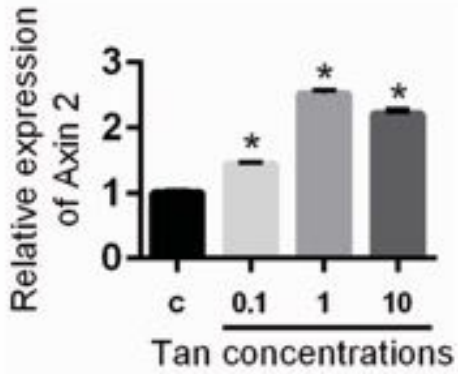

( $\mu \mathrm{M})$
Tan $10 \mu \mathrm{M}$

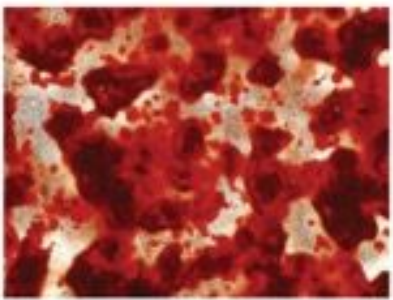

$\beta$-catenin

Collagen I

$\beta$-actin
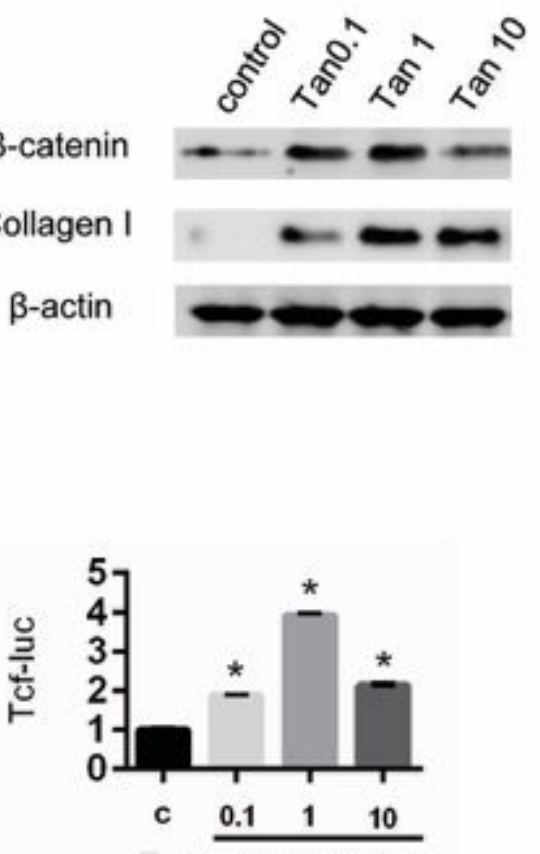

Tan concentrations

$(\mu \mathrm{M})$

Figure 1

Dose dependent effects of Tanshinol on osteogenesis of MSCs. Representative ARS staining images (a) at day 21. RT-PCR analysis of ALP (day 7), Collagen I gene expression (b) and $\beta$-catenin,Axin2 genes expression as well as the activity of Tcf transcriptional factors (d) in the four experimental groups examined at day 21. $\beta$-catenin and Collagen I gene expression normalized to $\beta$-actin (c) at day 21.Data are presented as mean $\pm S E M ; n=3$ individual experiments. ${ }^{*} p<0.05$ vs control. 
a

control

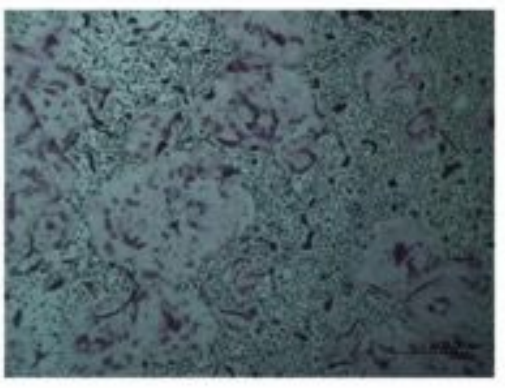

b

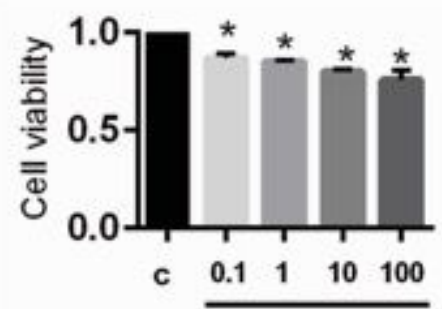

Tan concentrations

$(\mu \mathrm{M})$

Tan $25 \mu \mathrm{M}$

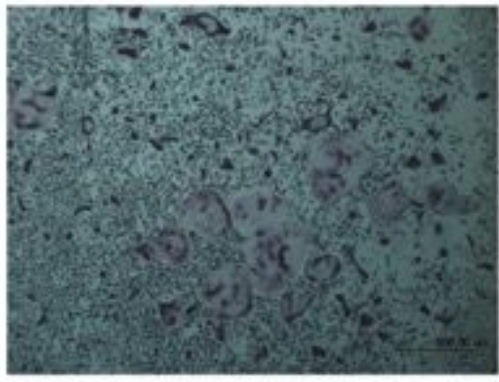

C

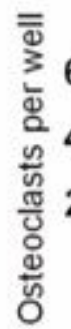

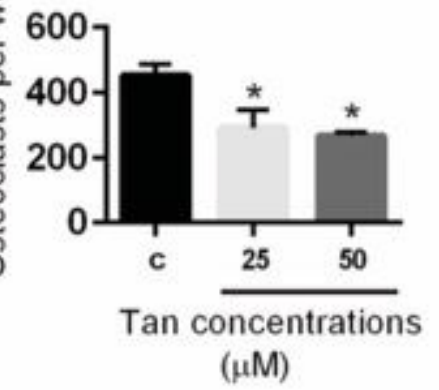

Tan $50 \mu \mathrm{M}$

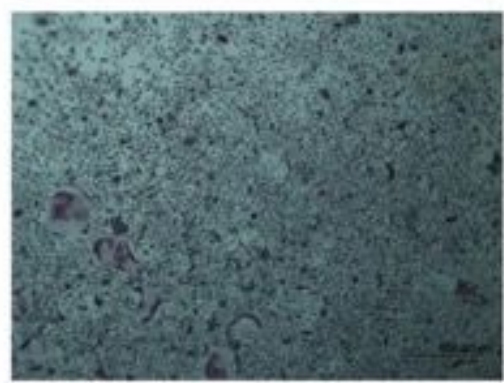

RANKL - + + +

Tan - $\quad 2550$

P-Akt

P-ERK

P-p38

P-JNK

$\beta$-actin

d
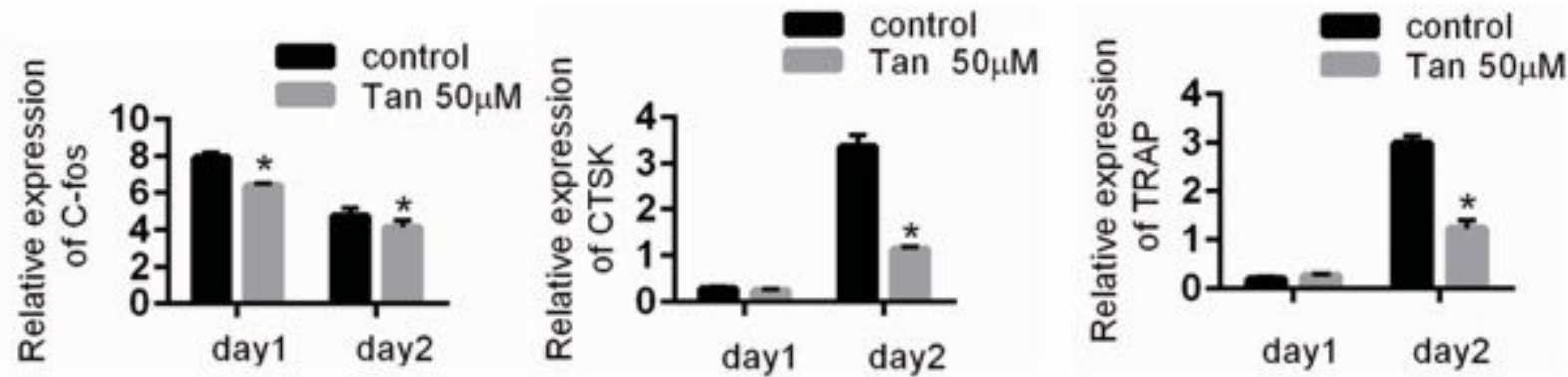

Figure 2

Tanshinol inhibits Osteoclastic differentiation of BMMs dose dependently. Tartrate resistant acid phosphatase (TRAP) staining (a) and cytotoxicity test (b).p-Akt, p-ERK, p38MAPK, and p-JNK genes expression normalized to $\beta$-actin (c). RT-PCR analysis of c-fos, CKST, NFATc1 (data were not shown) and TRAP genes expression (d). Data are presented as mean \pm SEM; $n=3$ individual experiments. ${ }^{*} p<0.05$ vs control. 
a

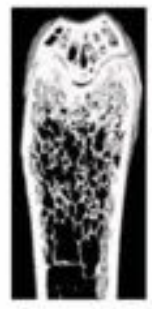

sham

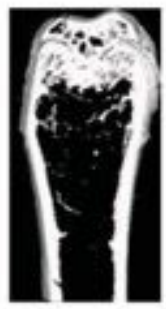

0

Ovx+ Tan concentrations $(\mathrm{mg} / \mathrm{kg} \times \mathrm{d})$

b

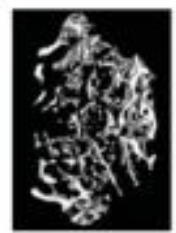

sham

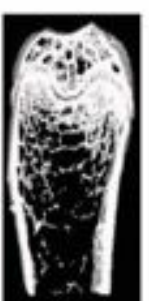

80

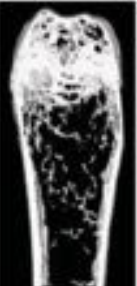

160

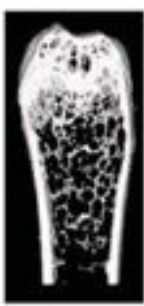

240

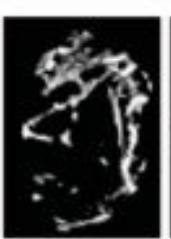

0

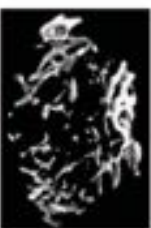

80

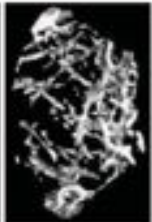

160

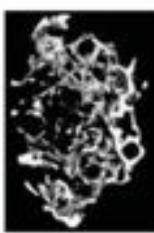

240

Ovx + Tan concentrations $(\mathrm{mg} / \mathrm{kg} \times \mathrm{d})$

c

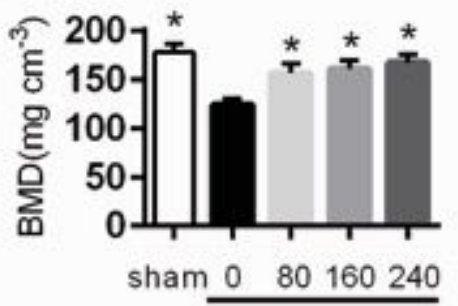

Tan concentrations

$(\mathrm{mg} / \mathrm{kg} \times \mathrm{d})$

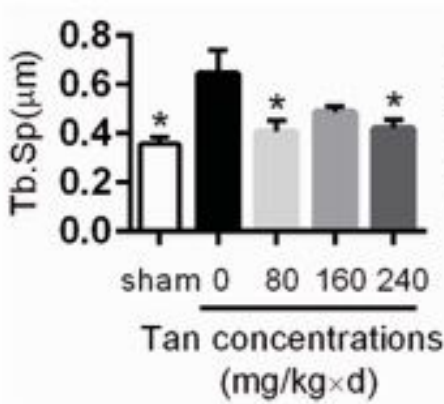

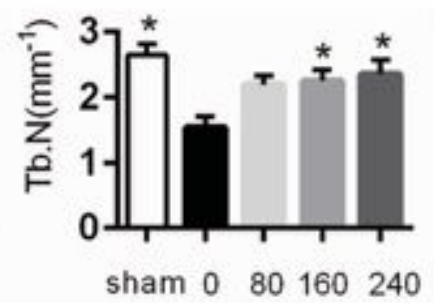

Tan concentrations $(\mathrm{mg} / \mathrm{kg} \times \mathrm{d})$

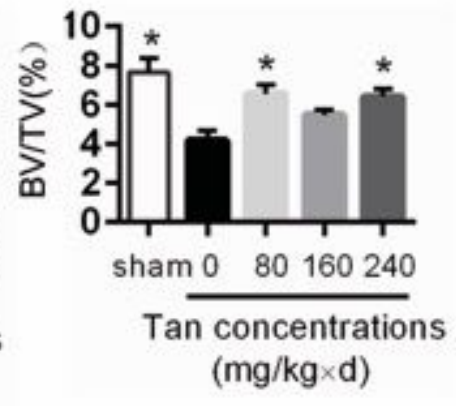

\section{Figure 3}

Tanshinol prevents the osteoporosis in the OVX mouse model. Representative 3D microarchitecture of the distal metaphysis of femur in each group $(n=6)$, obtained by microCT examination $(a$, and $b)$. BMD, Tb.N, Tb.Sp and BV/TV were detected by microCT examination of the mice in sham, OVX +free Tanshinol,

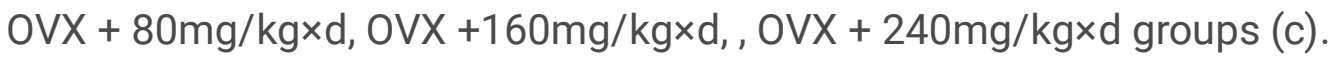

\section{Supplementary Files}

This is a list of supplementary files associated with this preprint. Click to download.

- Supplementarydata.docx 